\title{
Role of Radiographs and Ultrasound in Diagnosing Calcific Tendinitis and Periarthritis in the Wrist and Hand with Ultrasound-Guided Barbotage as Management Tool
}

\author{
Ankita Ahuja ${ }^{1,2}$ Malini Lawande ${ }^{1} \quad$ Aditya R. Daftary $^{1}$ \\ ${ }^{1}$ Innovision Imaging, Mumbai, Maharashtra, India \\ 2 Department of Radiodiagnosis, Tata Memorial Hospital, Mumbai, \\ Maharashtra, India \\ Indian J Radiol Imaging 2021;31:605-610.
}

Address for correspondence Ankita Ahuja, Department of Radiodiagnosis, Main Building, Tata Memorial Hospital, Parel, Mumbai 400014, Maharashtra, India (e-mail: ankita.ahuja2588@gmail.com).

\begin{abstract}
Keywords

- barbotage

- calcific

- periarthritis

- tendinitis

- ultrasound

Aim To demonstrate the role of radiographs and ultrasound (USG) in the diagnosis of calcific tendinitis and periarthritis in the wrist and hand and the efficacy of USG-guided barbotage for its management.

Materials and Methods A retrospective chart review was performed in six patients who presented with acute-onset pain in the wrist and hand varying from 3 days to 2 weeks. Four patients had tenderness over pisiform and two patients had pain along the lateral aspect of the wrist and thumb. Radiographs and USG revealed calcific focus corresponding to the site of pain. USG-guided calcific barbotage and injection was performed for the same and pain relief was assessed immediately and through telephonic follow-up at 6 months using subjective satisfaction score. Data were analyzed using Microsoft Excel 2013.

Results Four patients with tenderness over pisiform had flexor carpi ulnaris calcific tendinitis and two patients with pain along the lateral aspect of the wrist and thumb had first metacarpophalangeal calcific periarthritis and abductor pollicis brevis calcific tendinitis on radiographs as well as USG. In post-USG-guided calcific barbotage and injection, all patients had significant immediate and 6 months delayed relief in symptoms with excellent satisfaction scores.

Conclusion Acute calcific tendinitis/periarthritis is a benign and self-limiting inflammatory condition. Radiographs are extremely helpful in identifying calcific focus. Ultrasonography in experienced hands is the best modality to identify, confirm the symptomatic calcific focus, and perform USG-guided intervention. USG-guided calcific barbotage is the simplest, quickest, and effective way to treat this condition and avoid compromised functional capacity.
\end{abstract}

DOI https://doi.org/ 10.1055/s-0041-1736397. ISSN 0971-3026.
(C) 2021. Indian Radiological Association. All rights reserved. This is an open access article published by Thieme under the terms of the Creative Commons Attribution-NonDerivative-NonCommercial-License, permitting copying and reproduction so long as the original work is given appropriate credit. Contents may not be used for commercial purposes, or adapted, remixed, transformed or built upon. (https://creativecommons.org/ licenses/by-nc-nd/4.0/)

Thieme Medical and Scientific Publishers Pvt. Ltd., A-12, 2nd Floor, Sector 2, Noida-201301 UP, India 


\section{Background}

Acute calcific tendinitis/periarthritis is a benign and selflimiting inflammatory condition. It involves periarticular deposition of calcium hydroxyapatite crystals at variable locations including tendon, capsule, and ligaments. ${ }^{1}$ It predominantly affects people between 40 and 60 years age group with women being more often affected than men. ${ }^{2}$ It is most commonly described in the shoulder followed by hip. ${ }^{1,3}$ Involvement of the wrist and hand is less common. Strong clinical suspicion with radiographic evidence of calcification leads to correct diagnosis. ${ }^{4}$ Ultrasound (USG) and computed tomography (CT) demonstrate the calcification and help in confirmation of diagnosis. Magnetic resonance imaging (MRI) reveals focal edema in acute cases; however, small calcifications may not always be visualized on standard sequences. Three-dimensional gradient echo sequences are more sensitive in detecting the calcification. ${ }^{5}$ MRI and the clinical history also help exclude other differential possibilities such as infection and trauma.,

Being a self-limiting condition, a conservative approach is considered ideal for management. ${ }^{4,7,8}$ In some cases, however, there is excessive pain which impacts the quality of life. USG-guided needle barbotage including aspiration and injection is an effective treatment option. ${ }^{9}$ Radiation therapy and surgical excision are other less often used alternatives.

\section{Case Series}

\section{Materials and Methods}

A retrospective chart review was performed in six patients with acute onset of pain in the wrist and hand varying from 3 days to 2 weeks. All patients were examined clinically and radiologically with radiographs and USGs confirming the cause of pain as calcific tendinitis/periarthritis. All of them underwent USG-guided barbotage and injection for the same. Informed consent was obtained from all the patients prior to the procedure. Immediate postprocedure symptomatic relief was documented. All patients were explained to follow up in case of any complaints/recurrence of pain. Further, all the patients were telephonically followed up at 6 months to assess symptomatic relief with a subjective satisfaction score. Satis-

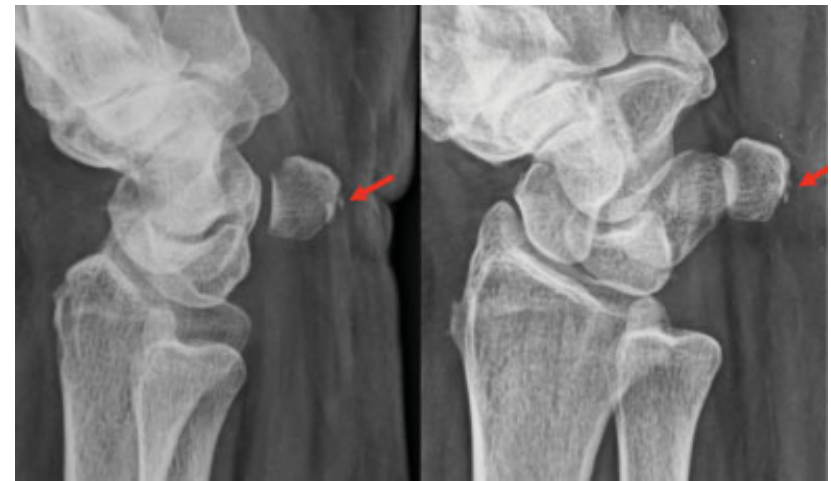

Fig. 1 Lateral and oblique radiographs of the wrist demonstrating a small focus of calcification on the ventral aspect of the pisiform bone (red arrow).

faction scores were recorded on a scale of 1 to 10 (1-4= poor, 5-7 = average, and 8-10=excellent). The data were analyzed retrospectively using Microsoft Excel 2013.

Four of these patients presented with acute-onset pain along the medial aspect of the wrist. On examination, they had localized tenderness over pisiform. In view of clinical suspicion of flexor carpi ulnaris calcific tendinitis, radiographic series including oblique views was obtained, which demonstrated calcifications in relation to the pisiform in all these patients (-Fig. 1). Subsequent USG confirmed calcifications in the flexor carpi ulnaris tendon near its pisiform attachment, which corresponded to the location of tenderness (- Figs. 2 and 3). Single needle technique, USG-guided barbotage, aspiration, and injection, was performed (-Fig. 4).

The other two patients presented with pain along the lateral aspect of the wrist and thumb. On examination, there was tenderness with restricted range of movements. Clinically, de Quervain's tenosynovitis and calcific tendinitis were the differential considerations. In the first patient, radiographs revealed slender calcification at the first metacarpophalangeal joint which was confirmed at USG and was adjoining the abductor pollicis brevis tendon, in keeping with calcific periarthritis ( - Fig. 5A, B). USG-guided barbotage was performed with significant immediate pain relief. Patient however had recurrent pain day after the procedure and a repeat USG examination revealed a small area of

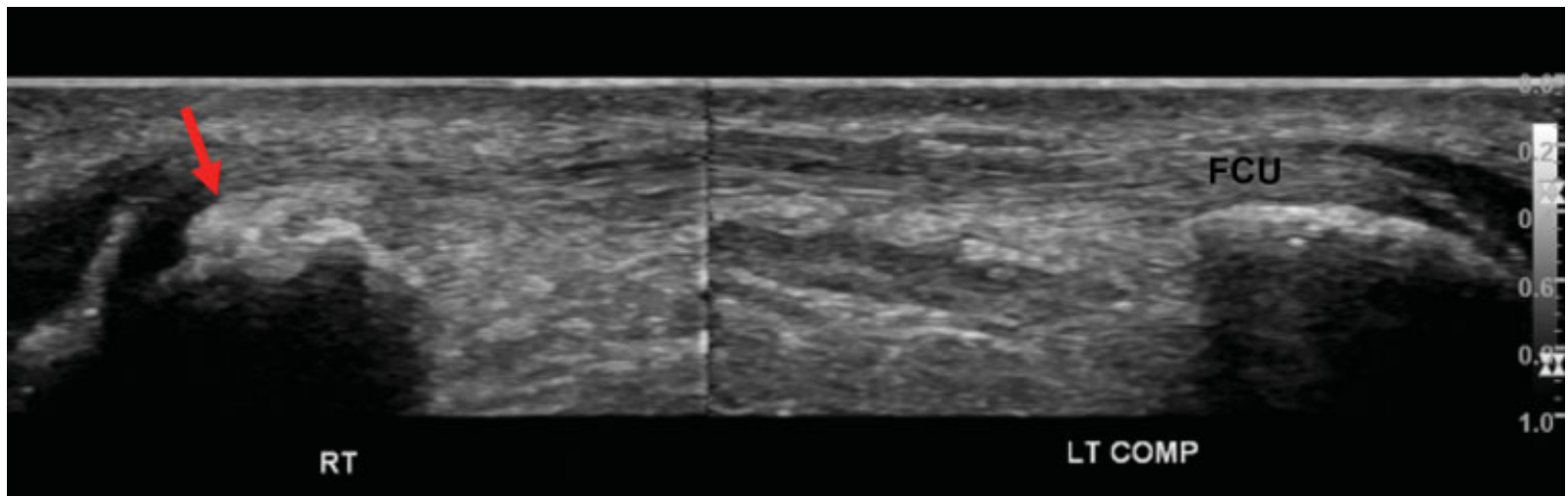

Fig. 2 Longitudinal ultrasound images of the wrist demonstrating calcific focus at the insertion of the right flexor carpi ulnaris (FCU) tendon on the right (red arrow) in contrast to the normal left FCU tendon. 


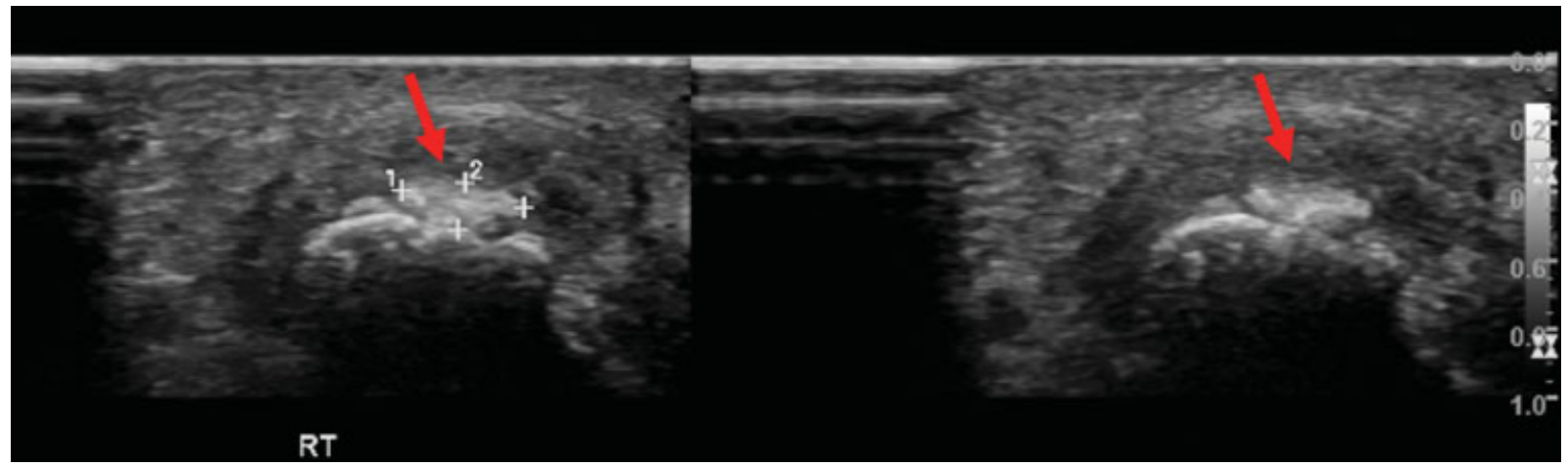

Fig. 3 Transverse ultrasound image of the wrist demonstrating calcific focus at the insertion of the right flexor carpi ulnaris tendon (red arrow).

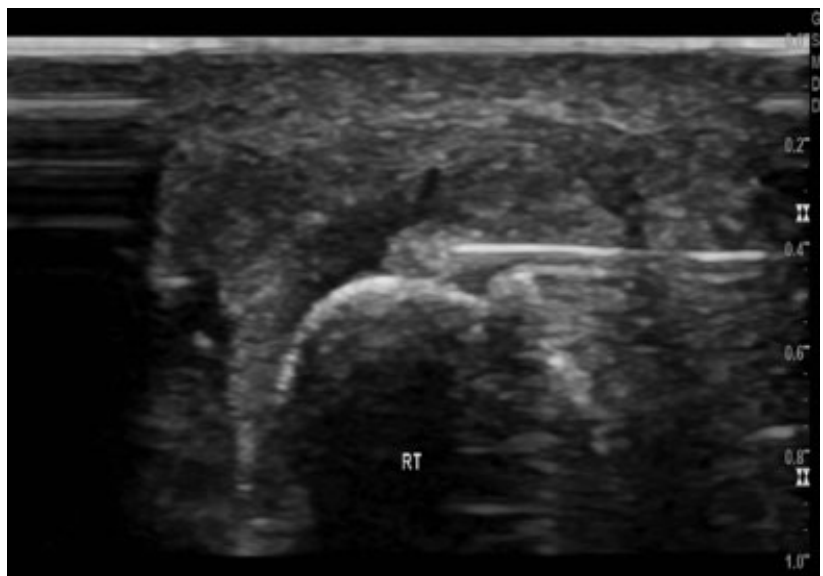

Fig. 4 Transverse ultrasound image of the wrist demonstrating needle within the flexor carpi ulnaris insertion calcific focus. persistent calcification which corresponded to the area of pain, and thus, the barbotage procedure was repeated. Retrospectively, it was felt that the first procedure may not have been completely successful due to air-related artifact which limited the visualization of calcification completely during the procedure.

The other patient had a history of de Quervain's tenosynovitis and had been treated with a steroid injection elsewhere. Radiographs and USG revealed two areas of soft calcification at the thumb base, which corresponded to the areas of localized tenderness in the carpometacarpal joint capsule, in keeping with acute calcific periarthritis (-Fig. 6). USG-guided barbotage was performed with significant symptomatic relief to the patient for the same.

\section{Technique}

USG-guided barbotage was performed following the administration of copious local anesthesia with $2 \%$ lignocaine under

A

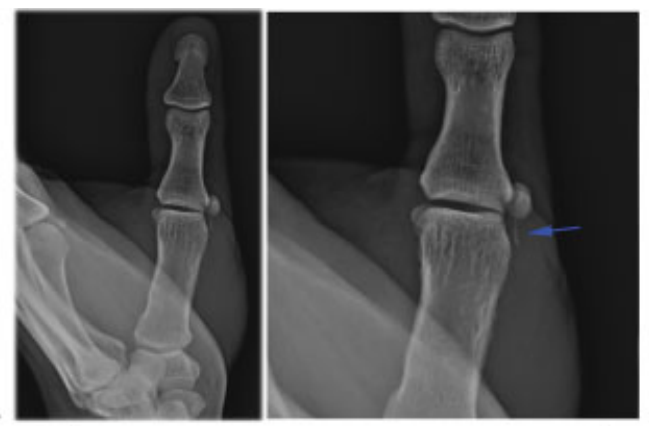

B
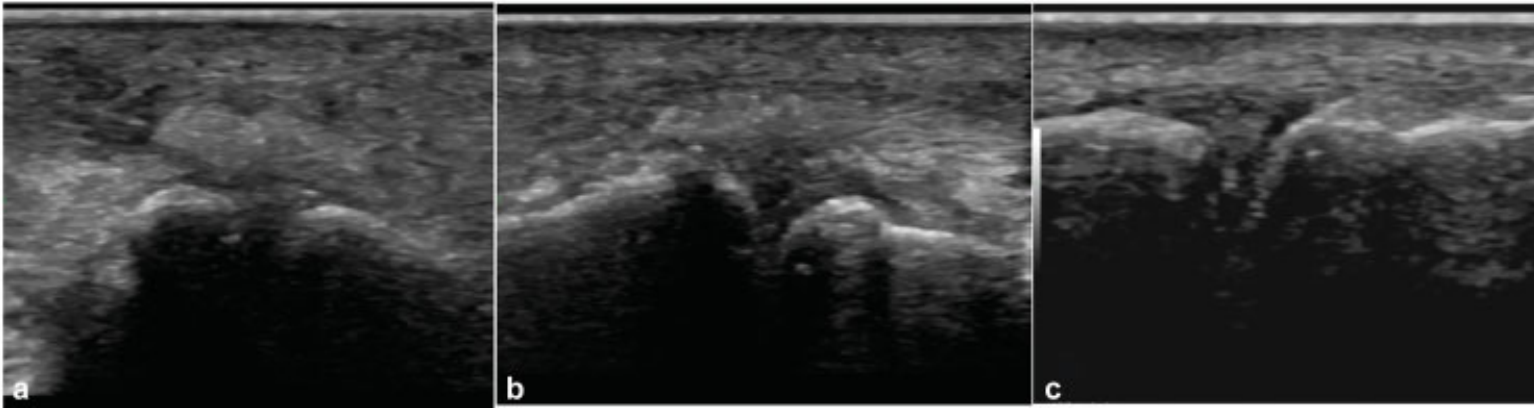

Fig. 5 (A) Frontal radiograph of the thumb with zoomed image on the left demonstrating thin slender calcification along the lateral aspect of the first metacarpophalangeal joint (blue arrow) (B) (a) Longitudinal ultrasound demonstrating calcific deposit at metacarpophalangeal joint, (b) immediate postbarbotage ultrasound demonstrating resolution of the calcific deposit, and (c) follow-up (>6 months) ultrasound demonstrating normal metacarpophalangeal joint without calcific deposit. 


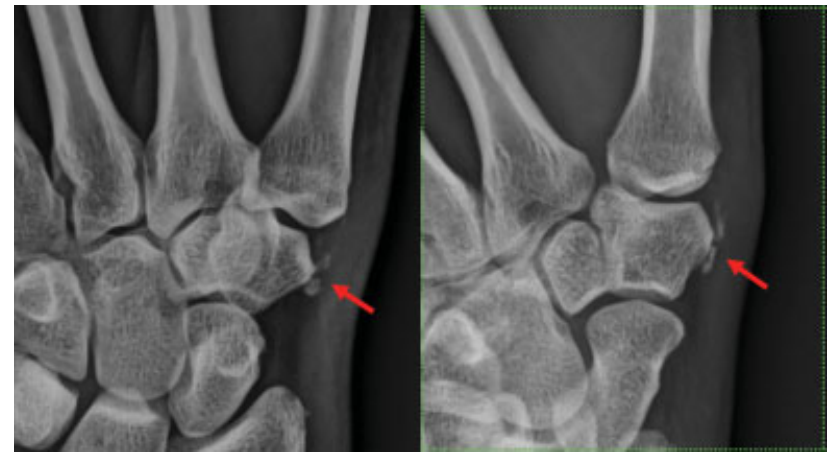

Fig. 6 Frontal and oblique radiographs of the wrist zoomed images demonstrating calcification along the lateral aspect of the carpometacarpal joint (red arrow).

USG guidance. A wider gauge needle (usually 18-22 gauge) was introduced into the calcification under direct USG guidance (-Fig. 4) with further barbotage/pulsing using normal saline, as a result of which calcium hydroxyapatite reacts with sodium chloride to form sodium hydroxyapatite and calcium chloride both of which were soluble and thus were aspirated. Subsequently, $0.5 \mathrm{~mL}$ each of $2 \%$ lignocaine and $0.5 \%$ bupivacaine as local anesthetic along with $20 \mathrm{mg}$ triamcinolone acetonide as steroid were injected in the adjacent tendon sheath, joint, bursa, or adjacent soft tissues as appropriate. Appropriate postinjection management with local icing was explained to the patient.

\section{Results}

All patients had marked immediate improvement of symptoms with excellent satisfaction scores $(>8 / 10)$. On 6 monthly telephonic follow-up, patient revealed persistent excellent ( $>8 / 10)$ subjective satisfaction scores with no significant complaints in the interim. No immediate or delayed complications were identified.

\section{Discussion}

Acute calcific tendinitis/periarthritis is a benign and selflimiting inflammatory condition, also known as peritendinitis calcarea, periarthritis calcarea, and calcium hydroxyapatite deposition disease. ${ }^{10}$

It was first described by Cohen in $1924 .{ }^{11}$ Case series by Carroll et al and Yelton reported 100 and more cases, thus indicating the frequency of occurrence of this entity. ${ }^{12}$ Since that time, there have been only case reports describing its occurrence sporadically in the literature. It is a well-known and common painful condition most often seen in the shoulder. ${ }^{1,3}$ The condition also occurs in multiple other locations all over the body but is often missed or misdiagnosed due to the lack of awareness. It has been described at many small joints of the hand and wrist involving both the flexor and extensor tendons, most common location being insertion of flexor carpi ulnaris tendon at pisiform. ${ }^{13}$

The etiology of the disease is unclear. The proposed and most convincing etiology is local stress necrosis which may occur as a result of mechanical (stress) or vascular insult and lead to transformation of tendon to fibrocartilage by activation of chondrocytic activity. The other etiologies include association with endocrine abnormalities and degeneration of the tendons/ligaments. ${ }^{6,14}$ Degenerative etiology seems unlikely as the peak age of incidence of calcific tendinitis tends to be earlier and it can resolve over time, in comparison to the degenerative tendinopathy which occurs in the later age and does not resolve. The chemical composition of deposited calcium salt is also different in both degenerative tendinopathy and calcific tendinitis. ${ }^{6,15}$

The current studies reveal it to be a cell-mediated process with four well-demarcated phases. (1) Formative: Fibrocartilaginous transformation of portion of the tendon. (2) Calcific: Calcium deposit enters a resting phase. It can be painful and can even cause mechanical symptoms if large. (3) Resorptive: Vascular tissue forms at the periphery, macrophages and multinuclear giant cells enter the area and lead to resorption of calcium. Calcium leaks out and causes inflammation. This is the most painful phase, which is when most patients present clinically. (4) Reparative: Fibroblasts restore the normal tendon collagen pattern. ${ }^{6}$ Uhthoff and Loehr ${ }^{4,14,16}$ described a three-phase process including precalcific, calcific, and postcalcific phases, of which calcific was further subdivided into three subphases as formative, resting, and resorptive.

The diagnosis of painful calcific periarthritis is usually in the resorptive phase. On radiographs, this presents as "soft" calcification. ${ }^{17,18}$ Smaller calcifications may be obscured in certain projections and be missed. Furthermore, determining the "hard" and "soft" phases of the calcification may also be a challenge at times. CT is more definitive in identifying and localizing calcifications but also has some of the same challenges as radiographs in determining phase of disease. The clinical relevance of a calcification is confirmed by subsequently correlating for localized tenderness.

USG is extremely useful as this not only identifies the calcification but it can also give good information on the soft or hard nature of the calcification as well as its phase from localized Doppler flow and probe pressure tenderness. ${ }^{18,19}$

MRI is often performed when the remaining techniques are inconclusive for the cause of pain. Currently, in some practices, it also ends up being the first investigation performed. Acute exacerbation of calcific periarthritis may present with extensive soft tissue edema (-Fig. 7) and the small calcification may

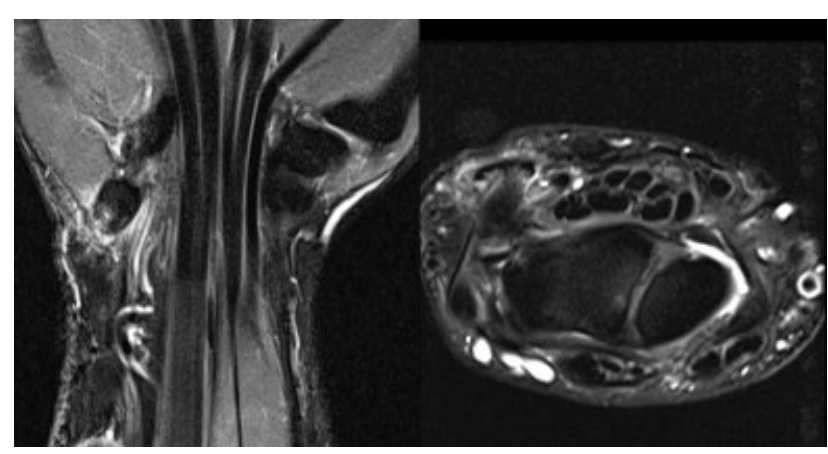

Fig. 7 Short tau inversion recovery coronal and axial images demonstrating edema at the insertion of the flexor carpi ulnaris and in the adjacent pisiform bone. 

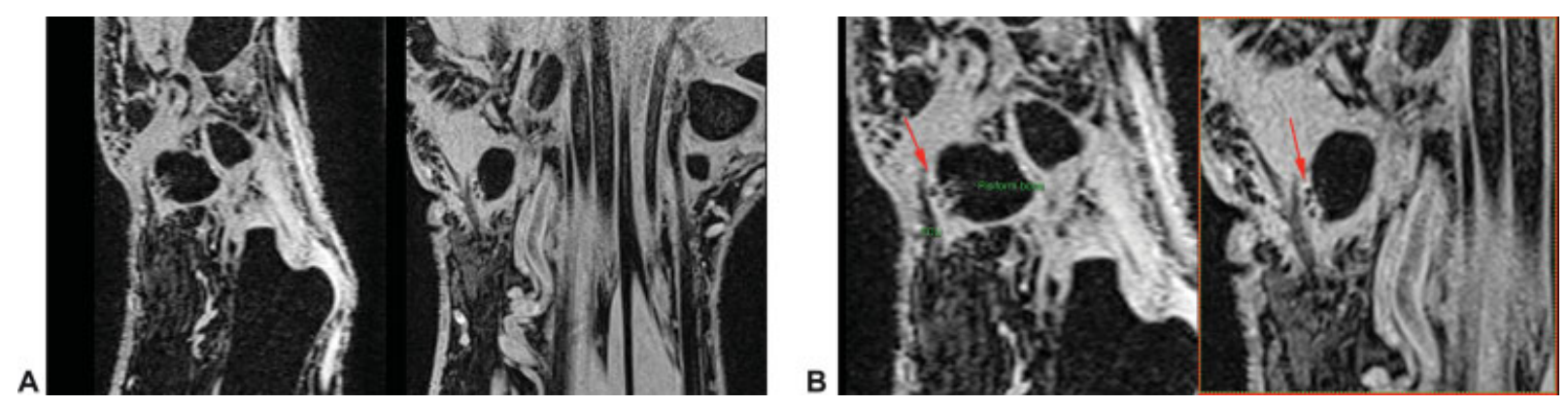

Fig. 8 (A) Gradient echo sagittal and coronal images demonstrating calcific foci at the insertion of the flexor carpi ulnaris (FCU) tendon. (B) Gradient echo sagittal and coronal zoomed images demonstrating calcific foci at the insertion of the FCU tendon (red arrow).

be hard to identify using standard imaging techniques. Gradient echo sequences ( - Fig. 8A, B) are usually most effective in identifying small calcifications., ${ }^{5,20}$ The absence of edema surrounding a calcification is also a good indicator that the calcification is not in the resorptive phase. It also helps exclude other pathologies responsible for similar kind of pain including septic arthritis/infection, osteomyelitis, trauma, tenosynovitis, or gout. 6,20

Calcific periarthritis resolves spontaneously over a period of a few weeks. A conservative approach with rest, antiinflammatory drugs, and local icing is usually considered to be sufficient for management. ${ }^{4,7,8,13,18}$ Persistent excruciating pain and motion limitation make the need for more aggressive therapy much more appropriate. USG-guided barbotage has been described extensively for its management in the shoulder, ${ }^{9,21}$ with case reports of successful management in other parts of the body. ${ }^{22}$ To the best of our knowledge, there are no case series specifically centered around the USG-guided barbotage of the wrist. In our series, all patients presented with extreme pain and underwent barbotage irrespective of the size of the calcification with excellent immediate and 6-month symptom relief demonstrating that USG and USG-guided barbotage are effective diagnostic and treatment options for calcific periarthritis around the wrist. Furthermore, USG can also demonstrate postprocedure reduction in the size and extent of calcification (-Fig. 5B). The relief of acute symptoms further prevents loss of working hours. Radiation therapy and surgical excision are other less often used alternatives.

There is varied information on the management by barbotage with saline lavage with or without steroid injection and steroid injection alone. Some authors also believe that smaller lesions do not need barbotage. However, in our study, one of the patient returned with persistent excruciating pain the next day and was localized to a small calcific area that had not been addressed at the time of the initial procedure suggesting barbotage may be a more appropriate course of action for even smaller calcifications around the wrist. Future prospective studies with and without steroids may be considered for further insight.

\section{Conclusion}

Radiographs and USG are the ideal and effective modalities to identify calcific periarthritis. USG is especially helpful to localize symptoms to the condition. USG-guided barbotage is an effective procedure in the management of this condition in and around the wrist. We highlight the presence of this condition in an uncommon location, the challenges with diagnosis and the apparent equal efficacy of treatment with barbotage in the wrist vis-à-vis other locations.

\section{Financial Support and Sponsorship}

Nil.

\section{Presentation at a Meeting}

This study was presented at the Musculoskeletal Society of India, Pune, August 24, 2019.

Conflict of Interest

None declared.

\section{References}

1 Hayes CW, Conway WF. Calcium hydroxyapatite deposition disease. Radiographics 1990;10(06):1031-1048

2 Bureau NJ. Calcific tendinopathy of the shoulder. Semin Musculoskelet Radiol 2013;17(01):80-84

3 Beckmann NM. Calcium apatite deposition disease: diagnosis and treatment. Radiol Res Pract 2016;2016:4801474

4 DE Carli A, Pulcinelli F, Rose GD, Pitino D, Ferretti A. Calcific tendinitis of the shoulder. Joints 2014;2(03):130-136

5 Nörenberg D, Ebersberger HU, Walter T, et al. Diagnosis of calcific tendonitis of the rotator cuff by using susceptibility-weighted MR imaging. Radiology 2016;278(02):475-484

6 Siegal DS, Wu JS, Newman JS, Del Cura JL, Hochman MG. Calcific tendinitis: a pictorial review. Can Assoc Radiol J 2009;60(05): 263-272

7 Cho NS, Lee BG, Rhee YG. Radiologic course of the calcific deposits in calcific tendinitis of the shoulder: does the initial radiologic aspect affect the final results? J Shoulder Elbow Surg 2010;19(02): 267-272

8 Vignesh KN, McDowall A, Simunovic N, Bhandari M, Choudur HN. Efficacy of ultrasound-guided percutaneous needle treatment of calcific tendinitis. AJR Am J Roentgenol 2015;204(01): 148-152

9 Gatt DL, Charalambous CP. Ultrasound-guided barbotage for calcific tendonitis of the shoulder: a systematic review including 908 patients. Arthroscopy 2014;30(09):1166-1172

10 Gandee RW, Harrison RB, Dee PM. Peritendinitis calcarea of flexor carpi ulnaris. AJR Am J Roentgenol 1979;133(06):1139-1141

11 Cohen I. Calcareous deposits at the insertion of flexor carpi ulnaris tendon following trauma. Am J Surg 1924;38:172-173

12 Carroll RE, Sinton W, Garcia A. Acute calcium deposits in the hand. J Am Med Assoc 1955;157(05):422-426 
Role of Radiographs and Ultrasound in Diagnosing Calcific Tendinitis and Periarthritis in the Wrist and Hand Ahuja

13 Harris AR, McNamara TR, Brault JS, Rizzo M. An unusual presentation of acute calcific tendinitis in the hand. Hand (N Y) 2009;4(01):81-83

14 Oliva F, Via AG, Maffulli N. Physiopathology of intratendinous calcific deposition. BMC Med 2012;10:95

15 Riley GP, Harrall RL, Constant CR, Cawston TE, Hazleman BL. Prevalence and possible pathological significance of calcium phosphate salt accumulation in tendon matrix degeneration. Ann Rheum Dis 1996;55(02):109-115

16 Uhthoff HK, Loehr JW. Calcific tendinopathy of the rotator cuff: Pathogenesis, diagnosis, and management. J Am Acad Orthop Surg 1997;5(04):183-191

17 Resnick DL, Kransdorf MJ. Bone and Joint Imaging E-Book. Elsevier Health Sciences; 2004

18 Chianca V, Albano D, Messina C, et al. Rotator cuff calcific tendinopathy: from diagnosis to treatment. Acta Biomed 2018; 89(1-S):186-196
19 Chiou HJ, Chou YH, Wu JJ, Hsu CC, Huang DY, Chang CY. Evaluation of calcific tendonitis of the rotator cuff: role of color Doppler ultrasonography. J Ultrasound Med 2002;21(03):289-295, quiz 296-297

20 Stoller DW, Tirman PF, Bredella MA, Beltram S, Branstetter RM, Blease S. Diagnostic Imaging Orthopaedics. 2004

21 Niazi G, Hetta W. The role of ultrasound guided percutaneous needle aspiration and lavage (barbotage) in the treatment of calcific tendinitis. Egypt J Radiol Nucl Med 2015; 46:63-70

22 McLoughlin E, Iqbal A, Tillman RM, James SL, Botchu R. Calcific tendinopathy of the direct head of rectus femoris: a rare cause of groin pain treated with ultrasound guided percutaneous irrigation. J Ultrasound 2020;23(03):425-430 